\title{
Lactoferrin Efficacy versus Ferrous Sulfate in Treatment of Children with Iron Deficiency Anemia
}

\author{
Osama Mahmoud El-Asheer ${ }^{10} \quad$ Ahmed Gaber Ahmed ${ }^{1} \quad$ Zainab AbdelAal Abdel Hafez ${ }^{2}$ \\ Marwa AbdelHafiz Dahpy ${ }^{3}$ Amal AbdEISalam Soliman ${ }^{1}$
}

\footnotetext{
1 Pediatric Department, Faculty of Medicine, Assiut University, Asyut, Egypt

${ }^{2}$ Clinical Pathology Department, Faculty of Medicine, Assiut University, Asyut, Egypt

${ }^{3}$ Department of Medical Biochemistry and Molecular Biology, Faculty of Medicine, Assiut University, Asyut, Egypt
}

J Child Sci 2021;11:e199-e204.

\author{
Address for correspondence Marwa AbdelHafiz Dahpy, Department \\ of Medical Biochemistry and Molecular Biology, Faculty of Medicine, \\ Assiut University, Asyut, Egypt \\ (e-mail: marwadahpy@yahoo.com; marwadahpy@aun.edu.eg).
}

\begin{abstract}
Keywords

- Iron

- iron deficiency anemia

- lactoferrin

- ferrous sulfate

- hemoglobin

Lactoferrin (LF) is an iron-binding globular glycoprotein that is structurally and chemically similar to serum transferrin. Many studies have been done to evaluate the effect of oral LF administration on iron deficiency anemia (IDA) with controversial results. This study was designed to compare the efficacy of LF versus oral ferrous sulfate (OFS) therapy in the treatment of children with IDA. A significant increase in mean hemoglobin and serum iron concentrations was noted in the group that received oral bovine $\operatorname{LF}(11.06 \pm 0.96$ and $42.79 \pm 6.14$, respectively) versus the group that received OFS ( $10.24 \pm 0.57$ and $28.94 \pm 5.05$, respectively, with $p<0.001$ for each) after 30 days of the treatment with fewer side effects ( 9.3 vs. $33.3 \%$ with $p=0.043$ ). Oral bovine LF is a more effective and safer alternative in treating iron deficiency and IDA compared with OFS with clinical benefits of fewer side effects and better patient compliance.
\end{abstract}

\section{Introduction}

Iron deficiency (ID) is the most widespread and common nutritional disorder in the world. It is estimated that 30 to $40 \%$ of the global population has ID anemia (IDA), and most of them live in developing countries. ${ }^{1}$ According to the 2001 World Health Organization (WHO) data, 30\% of the children aged between 0 and 4 years and $48 \%$ of the children aged between 5 and 14 years are anemic in developing countries. Egypt is well known to have the highest rates of IDA ( $>60 \%$ according to WHO) in addition to ID without anemia. ${ }^{1,2}$

The most common causes of IDA observed in children include inadequate intake, increased requirements with rapid growth together with low birth weight, decreased absorption, and gastrointestinal losses. ${ }^{2}$

received

December 7, 2020

accepted after revision

March 26, 2021
ID and IDA have many adverse effects on human health, including diminished mental, motor, and behavioral functioning that might not be completely reversible after treatment with iron. ${ }^{3}$

Oral ferrous sulfate (OFS) is the most commonly prescribed drug for treating ID; however, it has been associated with 25 to $40 \%$ incidence of adverse drug reactions (ADRs). Moreover, it has a variable bioavailability, thus stressing a need for better oral formulations. ${ }^{4}$

Lactoferrin (LF) is an iron-binding globular glycoprotein that is structurally and chemically similar to serum transferrin, the transporter of iron in the serum. It is widely distributed in all biological fluids and abundantly secreted in milk. It is produced by epithelial cells of the mucosa and is also expressed by immune cells that release it under stimulation by pathogens. ${ }^{5}$

(C) 2021. The Author(s).

This is an open access article published by Thieme under the terms of the Creative Commons Attribution License, permitting unrestricted use, distribution, and reproduction so long as the original work is properly cited. (https://creativecommons.org/licenses/by/4.0/)

Georg Thieme Verlag KG, Rüdigerstraße 14, 70469 Stuttgart, Germany 
LF is considered one of the components of the immune system of the body; it has bactericide and fungicide antimicrobial activity. LF interacts with DNA and RNA, polysaccharides and heparin, and shows some of its biological functions in complexes with these ligands. It acts as a mediator linking innate and adaptive immune responses. ${ }^{6}$ LF promotes iron absorption as it has 300 times higher affinity to iron as compared with serum transferrin. ${ }^{6}$ It maintains the balance of iron within the normal range and helps to avoid both ID and iron overload. Due to these reasons, LF is added to many commercial products of infant formulae. LF from bovine milk is the main LF used in human medicine because of its easy availability and has been designated by the United States Food and Drug Administration as a food additive that is generally recognized as safe. ${ }^{7}$

Many studies have been done to evaluate the effect of oral LF administration on IDA with controversial results.

A few among the numerous protective activities exercised by this protein, after its oral administration, are antianemic, anti-inflammatory, antimicrobial, immunomodulatory, antioxidant, and anticancer activities. ${ }^{7}$

These multiple significances in host defense and healthpromoting functions of LF and its wide real-life applications have stimulated increased research interest, which may play a role in solving global health problems such as IDA. ${ }^{8}$

\section{Method}

\section{Study Design and Setting}

The current work was designed as a randomized clinical trial study conducted over 10 months from January 2018 to October 2018 on 105 children attending Assiut University Children Hospital and known to have IDA to evaluate the effect of oral bovine LF, OFS, or combined therapy of both LF and OFS on treating IDA. Nine children were lost during the study, so a total of 96 children completed the study. They were randomly divided into three groups using a closed envelope containing a prewritten card with the group numbers I, II, or III.

Group I (LF group): 32 children received LF $100 \mathrm{mg}$ sachet over a quarter cup of water given once daily for 30 days on empty stomach.

Group II (iron group): 32 children received OFS (6 mg/ $/ \mathrm{kg} /$ day elemental iron) administered orally with meals, once daily for 30 days.

Group III (combination group): 32 children received both LF and iron for 30 days.

Inclusion criteria: the study included children above 2 years of age with IDA and both sexes were involved.

Exclusion criteria: children with one or more of the following (anemia rather than IDA, thalassemia trait, any family history of blood disease, any associated chronic illness or patients underwent iron therapy or received blood transfusions in the last 3 months) were excluded from this study.

Sample collection: $5 \mathrm{~mL}$ of blood was drawn from each child; $2 \mathrm{~mL}$ on a plain tube for serum collection and another
$3 \mathrm{~mL}$ on K3-EDTA vacutainer for complete blood count and reticulocyte count.

- All patients were subjected to full history and clinical examination. Pretherapy investigations included complete blood count, serum iron, serum ferritin, and stool analysis to exclude any parasitic infestation.

- Complete blood count was performed by automated ABX Pentra XL80 HORIBA (ABX-France). The complete blood count included red blood cell (RBC) count, hemoglobin $(\mathrm{Hb})$ concentration, mean corpuscular volume (MCV), mean corpuscular hemoglobin (MCH), $\mathrm{MCH}$ concentration (MCHC), and hematocrit (HCT) value.

- Serum ferritin was measured using Elisa kits by Invitrogen, (Thermo Fisher scientific, United States; Cat no \# EHFTL).

- Serum iron was measured using colorimetric assay, (Bio diagnostic, Egypt; Cat no \# IR 15 10).

- A 30-day treatment regimen and analysis of posttherapy investigations (complete blood count and serum iron) were done, which evaluated the efficacy of the treatment given on hemoglobin concentration and serum iron level.

\section{Statistical Analysis}

The data were tested for normality using the Anderson-Darling test and for homogeneity variances before further statistical analysis. Categorical variables were described by number and percent $(n, \%)$, where continuous variables were described by the mean and standard deviation (mean, SD). Chi-squared test and Fisher's exact test were used to compare categorical variables while comparisons between continuous variables were examined by $t$-test and ANOVA (analysis of variance). A two-tailed $p<0.05$ was considered statistically significant. All analyses were performed with the IBM SPSS 20.0 software.

\section{Results}

In this present study, we included children above 2 years of age with IDA. Their ages ranged from 2 to 15 years with a mean age $\pm S D$ of $5.12 \pm 2.58$ years. The number of children aged 2 to 6 years was 55 (57.3\%); however, those $\geq 6$ were 41 (42.7\%). Of the studied children, $77.1 \%$ were from rural areas and $22.9 \%$ were from urban areas. Both sexes were involved; there were 54 male children (56.2\%) and 42 females (43.7\%). A study of the anthropometric measures of studied cases $(n=96)$ showed that $68.8 \%$ had normal weight for age while $31.2 \%$ had low weight for age, and $55.2 \%$ had normal height for age and $44.8 \%$ were stunted. Comparing the biochemical findings (serum iron and serum ferritin) and hematological finding of complete blood count before the start of therapy showed no significant differences in baseline values between the three included groups (-Table $\mathbf{1}$ ).

Complete blood count and iron status before and after LF therapy and ferrous sulfate therapy are shown in - Tables 2 and $\mathbf{3}$, respectively. A comparison of the results before and after therapy showed that there is a statistically significant increase in $\mathrm{RBC}$ count, $\mathrm{Hb}, \mathrm{HCT}, \mathrm{MCV}, \mathrm{MCH}$, and serum iron after treatment in the three groups compared with baseline (-Table 2). There is a statistically significant difference in the hematological parameters RBCs, Hb, HCT, MCV, MCH, and 
Table 1 Hematological and biochemical parameters in the three studied groups before therapy

\begin{tabular}{|c|c|c|c|c|c|c|c|}
\hline \multirow[t]{2}{*}{ Variable } & \multicolumn{7}{|l|}{ Before therapy } \\
\hline & Group I & Group II & Group III & $p$-Value & P1 & P2 & P3 \\
\hline WBC $\left(10^{3} / \mu \mathrm{L}\right)$ & $8.93 \pm 2.03$ & $9.58 \pm 2.22$ & $9.52 \pm 1.88$ & 0.374 & 0.200 & 0.258 & 0.894 \\
\hline RBCs $\left(10^{6} / \mu \mathrm{L}\right)$ & $4.46 \pm 0.47$ & $4.24 \pm 0.42$ & $4.25 \pm 0.65$ & 0.168 & 0.091 & 0.116 & 0.925 \\
\hline $\mathrm{Hb}(\mathrm{g} / \mathrm{dL})$ & $9.95 \pm 0.87$ & $9.95 \pm 0.56$ & $10.05 \pm 0.75$ & 0.806 & 0.994 & 0.573 & 0.564 \\
\hline PLt $\left(10^{3} / \mathrm{mm}^{3}\right)$ & $315.44 \pm 27.14$ & $312.58 \pm 57.11$ & $323.19 \pm 86.52$ & 0.777 & 0.852 & 0.618 & 0.492 \\
\hline $\mathrm{MCV}(\mathrm{fL})$ & $70.22 \pm 2.46$ & $69.15 \pm 3.28$ & $70.81 \pm 4.17$ & 0.139 & 0.204 & 0.485 & 0.051 \\
\hline $\mathrm{MCH}(\mathrm{pg})$ & $22.63 \pm 1.93$ & $23.42 \pm 1$ & $22.89 \pm 1.3$ & 0.091 & $0.032^{*}$ & 0.483 & 0.152 \\
\hline $\mathrm{MCHC}(\mathrm{g} / \mathrm{dL})$ & $32.36 \pm 1.27$ & $32.52 \pm 1.23$ & $32.27 \pm 1.33$ & 0.741 & 0.624 & 0.784 & 0.447 \\
\hline $\mathrm{HCT}(\%)$ & $30.79 \pm 3.09$ & $30.55 \pm 3.15$ & $31.31 \pm 2.69$ & 0.584 & 0.742 & 0.492 & 0.309 \\
\hline RDW (fL) & $14.88 \pm 1.92$ & $15.46 \pm 1.93$ & $14.7 \pm 1.54$ & 0.219 & 0.199 & 0.702 & 0.098 \\
\hline Ret\% & $0.63 \pm 0.11$ & $0.65 \pm 0.13$ & $0.63 \pm 0.12$ & 0.787 & 0.577 & 0.941 & 0.530 \\
\hline Serum iron $(\mu \mathrm{g} / \mathrm{dL})$ & $24.17 \pm 2.67$ & $22.88 \pm 4.91$ & $24.09 \pm 3.55$ & 0.323 & 0.180 & 0.933 & 0.213 \\
\hline Serum ferritin $(\mathrm{ng} / \mathrm{mL})$ & $8.54 \pm 2.63$ & $9.3 \pm 2.21$ & $8.84 \pm 2.57$ & 0.460 & 0.210 & 0.640 & 0.440 \\
\hline
\end{tabular}

Abbreviations: $\mathrm{Hb}$, hemoglobin; $\mathrm{HCT}$, hematocrit; $\mathrm{MCH}$, mean corpuscular hemoglobin; $\mathrm{MCHC}$, mean corpuscular hemoglobin concentration; MCV, mean corpuscular volume; PLt, platelet; RBC, red blood cell; Ret, reticulocyte; WBC, white blood cell.

Note: The results were expressed as mean \pm SD. ANOVA test was used to compare different markers between the three groups.

*Statistically significant. p-value: comparison between all groups (I, II and III); P1: comparison between I and II, P2: comparison between I and III; P3: comparison between II and III.

serum iron after treatment in group I and group III compared with group II. However, there were no statistically significant differences in these hematological parameters after treatment between group I and group III ( - Table 3 ).

To test the percentage of improvement of anemia (increase of hemoglobin level) after therapy, our data showed that there is a significant difference in percentage of improvement of anemia after therapy between group I (53.1\%) and group II $(21.2 \%)(p<0.05)$, and significant difference between group III (61.3\%) and group II $(21.2 \%)(p<0.01)$; however, there is no significant difference in percentage of improvement of anemia after therapy between group I (53.1\%) and group III (61.3\%) $(p>0.05)$.

After the 30-day therapy, we documented the adverse effects (ADRs) for both LF therapy and ferrous sulfate therapy amongst the three groups; results show that there is a significant difference among the three included groups ( $p<0.05$ each), while the lowest adverse effects were noticed in group I after LF therapy (-Table 4). The data from our study showed statistically significant difference of adverse effects between group I (9.3\%), group II (33.3\%), and group III $(16.1 \%)(p<0.05)$. Cases who were administered oral bovine LF reported fewer adverse effects than those on OFS.

The data showed a significant increase in compliance of group I (93.7\%) compared with group II $(54.5 \%)(p<0.001)$; also there was a significant increase in compliance of group III (90.3\%) compared with group II $(54.5 \%)(p<0.01)$, but there was no significant difference in compliance between group I (93.7\%) and group III (90.3\%) ( $p>0.05)$.

\section{Discussion}

ID affects more than 2 billion people globally, with greater prevalence in women and children. In this present study the age distribution of population was from 2 to 15 years as they are most vulnerable to IDA. Our results were in accordance with that of Russo et al, who stated that after the second year of life the iron requirement rapidly increases, and diet becomes a critical issue. ${ }^{9}$ OFS is the most prescribed drug for treating ID; however, it has been associated with 25 to $40 \%$ incidence of ADRs. Moreover, it has a variable bioavailability, thus stressing a need for better oral formulations. ${ }^{4} \mathrm{LF}$ is an iron-binding glycoprotein that is structurally and chemically similar to serum transferrin, the transporter of iron in the serum. ${ }^{5}$ LF promotes iron absorption as it has 300 times higher affinity to iron as compared with serum transferrin. ${ }^{6}$ The serum ferritin level is well known to be the best parameter for reflecting iron status in patients with IDA, but it needs 3 months of treatment for replenishing iron stores and to be re-evaluated after treatment. ${ }^{8}$ In our study, serum iron, $\mathrm{Hb}$ level, and absolute reticulocyte count (ARC) were taken as parameters of improvement due to the short period of follow-up allowed in our study protocol (1 month). Adamson also stated that serum iron is the parameter that can be corrected early after treatment and reflecting a good response to treatment, while at least a 3-month course of therapy is required for restoring iron stores in the body and normalization of serum ferritin level. ${ }^{10}$

Our study showed a significant increase in $\mathrm{Hb}$ level and serum iron concentration in group I (receiving oral bovine LF) and group III (receiving combination therapy) compared with group II (receiving OFS). This was in agreement with the results of Russo et al, who compared the efficacy of different iron preparations, by the increase in Hb concentration in comparison with basal level.

Also, El-Khawaga and Abdelmaksoud studied primary school children who received LF $100 \mathrm{mg}$ twice daily for 30 days, which reported a significant increase in the mean 
Table 2 Hematological and biochemical parameters before and after Lactoferrin, Ferrous sulfate and combined therapy

\begin{tabular}{|c|c|c|c|c|}
\hline \multirow[t]{2}{*}{ Variable } & \multicolumn{4}{|c|}{ Lactoferrin (I) (n=32) } \\
\hline & Before & After & Mean difference & $\mathrm{p}$-Value \\
\hline WBC $\left(10^{3} / \mu \mathrm{L}\right)$ & $8.93 \pm 2.03$ & $7.57 \pm 1.64$ & -1.36 & $0.005^{*}$ \\
\hline $\operatorname{RBC}\left(10^{6} / \mu \mathrm{L}\right)$ & $4.46 \pm 0.47$ & $4.69 \pm 0.19$ & 0.23 & $0.011^{*}$ \\
\hline $\mathrm{Hb}(\mathrm{g} / \mathrm{dL})$ & $9.95 \pm 0.87$ & $11.06 \pm 0.96$ & 1.11 & $<0.001^{*}$ \\
\hline PLt $\left(10^{3} / \mathrm{mm}^{3}\right)$ & $315.44 \pm 27.14$ & $306.41 \pm 46.98$ & -9.03 & 0.350 \\
\hline $\mathrm{MCV}(\mathrm{fL})$ & $70.22 \pm 2.46$ & $74.59 \pm 2.5$ & 4.37 & $<0.001^{*}$ \\
\hline $\mathrm{MCH}(\mathrm{pg})$ & $22.63 \pm 1.93$ & $25.05 \pm 1.59$ & 2.42 & $<0.001^{*}$ \\
\hline $\mathrm{MCHC}(\mathrm{g} / \mathrm{dL})$ & $32.36 \pm 1.27$ & $32.39 \pm 1.39$ & 0.03 & 0.940 \\
\hline HCT (\%) & $30.79 \pm 3.09$ & $34.2 \pm 1.69$ & 3.41 & $<0.001^{*}$ \\
\hline RDW (fL) & $14.88 \pm 1.92$ & $12.74 \pm 1.4$ & -2.14 & $<0.001^{*}$ \\
\hline Ret\% & $0.63 \pm 0.11$ & $1.91 \pm 0.5$ & 1.28 & $<0.001^{*}$ \\
\hline Serum iron $(\mu \mathrm{g} / \mathrm{dL})$ & $24.17 \pm 2.67$ & $42.79 \pm 6.14$ & 18.62 & $<0.001^{*}$ \\
\hline \multirow[t]{2}{*}{ Variable } & \multicolumn{4}{|c|}{ Ferrous sulfate therapy (III) $(n=32)$} \\
\hline & Before & After & Mean difference & $p$-Value \\
\hline WBC $\left(10^{3} / \mu \mathrm{L}\right)$ & $9.58 \pm 2.22$ & $8.62 \pm 1.53$ & -0.96 & $0.043^{*}$ \\
\hline $\operatorname{RBC}\left(10^{6} / \mu \mathrm{L}\right)$ & $4.24 \pm 0.42$ & $4.43 \pm 0.34$ & 0.19 & $0.043^{*}$ \\
\hline $\mathrm{Hb}(\mathrm{g} / \mathrm{dL})$ & $9.95 \pm 0.56$ & $10.24 \pm 0.57$ & 0.29 & $0.041^{*}$ \\
\hline PLt $\left(10^{3} / \mathrm{mm}^{3}\right)$ & $312.58 \pm 57.11$ & $304.21 \pm 34.03$ & -8.37 & 0.472 \\
\hline $\mathrm{MCV}(\mathrm{fL})$ & $69.15 \pm 3.28$ & $72.88 \pm 1.7$ & 3.73 & $<0.001^{*}$ \\
\hline $\mathrm{MCH}(\mathrm{pg})$ & $23.42 \pm 1$ & $23.9 \pm 1.42$ & 0.48 & $0.048^{*}$ \\
\hline $\mathrm{MCHC}(\mathrm{g} / \mathrm{dL})$ & $32.52 \pm 1.23$ & $32.55 \pm 1.31$ & 0.03 & 0.908 \\
\hline HCT (\%) & $30.55 \pm 3.15$ & $32.78 \pm 2.8$ & 2.23 & $0.034^{*}$ \\
\hline RDW (fL) & $15.46 \pm 1.93$ & $14.3 \pm 1.28$ & -1.16 & $0.006^{*}$ \\
\hline Ret\% & $0.65 \pm 0.13$ & $1.65 \pm 0.52$ & 1 & $<0.001^{*}$ \\
\hline Serum iron $(\mu \mathrm{g} / \mathrm{dL})$ & $22.84 \pm 4.10$ & $28.94 \pm 5.05$ & 6.06 & $<0.001^{*}$ \\
\hline \multirow[t]{2}{*}{ Variable } & \multicolumn{4}{|c|}{ Combined (III) $(n=32)$} \\
\hline & Before & After & Mean difference & p-Value \\
\hline WBC $\left(10^{3} / \mu \mathrm{L}\right)$ & $9.52 \pm 1.88$ & $8.25 \pm 1.58$ & -1.27 & $0.006^{*}$ \\
\hline $\operatorname{RBC}\left(10^{6} / \mu \mathrm{L}\right)$ & $4.25 \pm 0.65$ & $4.75 \pm 0.39$ & 0.5 & $<0.001^{*}$ \\
\hline $\mathrm{Hb}(\mathrm{g} / \mathrm{dL})$ & $10.05 \pm 0.75$ & $11.24 \pm 0.71$ & 1.19 & $<0.001^{*}$ \\
\hline PLt $\left(10^{3} / \mathrm{mm}^{3}\right)$ & $323.19 \pm 86.52$ & $328.1 \pm 65.41$ & 4.91 & 0.802 \\
\hline $\mathrm{MCV}(\mathrm{fL})$ & $70.81 \pm 4.17$ & $74.18 \pm 1.99$ & 3.37 & $<0.001^{*}$ \\
\hline $\mathrm{MCH}(\mathrm{pg})$ & $22.89 \pm 1.3$ & $25.17 \pm 1.67$ & 2.28 & $<0.001^{*}$ \\
\hline $\mathrm{MCHC}(\mathrm{g} / \mathrm{dL})$ & $32.27 \pm 1.33$ & $32.52 \pm 1.43$ & 0.25 & 0.481 \\
\hline $\mathrm{HCT}(\%)$ & $31.31 \pm 2.69$ & $34.72 \pm 1.31$ & 3.41 & $<0.001^{*}$ \\
\hline RDW (fL) & $14.7 \pm 1.54$ & $12.32 \pm 1.3$ & -2.38 & $<0.001^{*}$ \\
\hline Ret\%. & $0.63 \pm 0.12$ & $2.06 \pm 0.25$ & 1.43 & $<0.001^{*}$ \\
\hline Serum iron $(\mu \mathrm{g} / \mathrm{dL})$ & $24.09 \pm 3.55$ & $45.67 \pm 8.42$ & 21.58 & $<0.001^{*}$ \\
\hline
\end{tabular}

Abbreviations: $\mathrm{Hb}$, hemoglobin; $\mathrm{HCT}$, hematocrit; $\mathrm{MCH}$, mean corpuscular hemoglobin; $\mathrm{MCHC}$, mean corpuscular hemoglobin concentration; $\mathrm{MCV}$, mean corpuscular volume; PLt, platelet; RBC, red blood cell; Ret, reticulocyte; WBC, white blood cell.

Note: The results were expressed as mean \pm SD. $t$-test was used to compare different markers between each group before and after therapy.

*Statistically significant.

$\mathrm{Hb}$ level and serum iron concentration. The mean rises in $\mathrm{Hb}$ and serum iron concentration in their study were higher than those recorded in our study and this can be explained by higher doses of LF used in their study. ${ }^{11}$ Similar results were also detected among Indian females aged 18 to 21 years, ${ }^{12}$ where a significant increase of $\mathrm{Hb}$ level and serum iron was reported in cases that received LF fortified bovine colostrum once daily for 30 days and in cases that received combined therapy. 
Table 3 Comparison between three groups after therapy regarding hematological findings and serum iron

\begin{tabular}{|l|l|l|l|l|l|l|l|}
\hline \multirow{2}{*}{ Variable } & \multicolumn{7}{l|}{ After therapy } \\
\cline { 2 - 8 } & Group I & Group II & Group III & $p$-Value & P1 & P2 & P3 \\
\hline WBC $\left(10^{3} / \mu \mathrm{L}\right)$ & $7.57 \pm 1.64$ & $8.62 \pm 1.53$ & $8.25 \pm 1.58$ & $0.030^{\mathrm{a}}$ & $0.009^{\mathrm{b}}$ & 0.095 & 0.349 \\
\hline RBC $\left(10^{6} / \mu \mathrm{L}\right)$ & $4.69 \pm 0.19$ & $4.43 \pm 0.34$ & $4.75 \pm 0.39$ & $<0.001^{\mathrm{b}}$ & $0.001^{\mathrm{b}}$ & 0.519 & $<0.001^{\mathrm{b}}$ \\
\hline $\mathrm{Hb}(\mathrm{g} / \mathrm{dL})$ & $11.06 \pm 0.96$ & $10.24 \pm 0.57$ & $11.24 \pm 0.71$ & $<0.001^{\mathrm{b}}$ & $<0.001^{\mathrm{b}}$ & 0.368 & $<0.001^{\mathrm{b}}$ \\
\hline $\mathrm{PLt}\left(10^{3} / \mathrm{mm}^{3}\right)$ & $306.41 \pm 46.98$ & $304.21 \pm 34.03$ & $328.1 \pm 65.41$ & 0.118 & 0.860 & 0.089 & 0.060 \\
\hline $\mathrm{MCV}(\mathrm{fL})$ & $74.59 \pm 2.5$ & $72.88 \pm 1.7$ & $74.18 \pm 1.99$ & $0.004^{\mathrm{b}}$ & $0.001^{\mathrm{b}}$ & 0.445 & $0.014^{\mathrm{a}}$ \\
\hline $\mathrm{MCH}(\mathrm{pg})$ & $25.05 \pm 1.59$ & $23.9 \pm 1.42$ & $25.17 \pm 1.67$ & $<0.001^{\mathrm{b}}$ & $<0.001^{\mathrm{b}}$ & 0.760 & $0.011^{\mathrm{a}}$ \\
\hline $\mathrm{MCHC}(\mathrm{g} / \mathrm{dL})$ & $32.39 \pm 1.39$ & $32.55 \pm 1.31$ & $32.52 \pm 1.43$ & 0.875 & 0.626 & 0.698 & 0.926 \\
\hline $\mathrm{HCT}(\%)$ & $34.2 \pm 1.69$ & $32.78 \pm 2.8$ & $34.72 \pm 1.31$ & $<0.001^{\mathrm{b}}$ & $0.015^{\mathrm{a}}$ & 0.170 & $<0.001^{\mathrm{b}}$ \\
\hline RDW $(\mathrm{fL})$ & $12.74 \pm 1.4$ & $14.3 \pm 1.28$ & $12.32 \pm 1.3$ & $<0.001^{\mathrm{b}}$ & $<0.001^{\mathrm{b}}$ & 0.209 & $<0.001^{\mathrm{b}}$ \\
\hline Ret\% & $1.91 \pm 0.5$ & $1.65 \pm 0.52$ & $2.06 \pm 0.25$ & $0.001^{\mathrm{b}}$ & $0.017^{\mathrm{a}}$ & 0.177 & $<0.001^{\mathrm{b}}$ \\
\hline Serum iron $(\mu \mathrm{g} / \mathrm{dL})$ & $42.79 \pm 6.14$ & $28.94 \pm 5.05$ & $45.67 \pm 8.42$ & $<0.001^{\mathrm{b}}$ & $<0.001^{\mathrm{b}}$ & 0.089 & $<0.001^{\mathrm{b}}$ \\
\hline
\end{tabular}

Abbreviations: $\mathrm{Hb}$, hemoglobin; $\mathrm{HCT}$, hematocrit; $\mathrm{MCH}$, mean corpuscular hemoglobin; MCHC, mean corpuscular hemoglobin concentration; MCV, mean corpuscular volume; PLt, platelet; RBC, red blood cell; Ret, reticulocyte; WBC, white blood cell.

Note: The results were expressed as mean \pm SD. ANOVA test was used to compare different markers between the three groups. $p<0.05$ was considered statistically significant.

aStatistically significant. p-Value: comparison between all groups (I, II, and III), P1: comparison between I and II; P2: comparison between I and III; P3: comparison between II and III.

Table 4 Adverse drug reactions among the three studied groups.

\begin{tabular}{|c|l|l|l|l|l|l|l|l|l|l|}
\hline & $\begin{array}{l}\text { Group I } \\
(\boldsymbol{n}=32)\end{array}$ & $\begin{array}{l}\text { Group II } \\
(\boldsymbol{n}=33)\end{array}$ & \multicolumn{2}{l|}{$\begin{array}{l}\text { Group III } \\
(\boldsymbol{n}=31)\end{array}$} & \multicolumn{3}{l|}{$p$-Value } \\
\cline { 2 - 6 } & No & $\%$ & No & $\%$ & No & $\%$ & All & P1 & P2 & P3 \\
\hline Adverse effects & 3 & 9.3 & 11 & 33.3 & 5 & 16.1 & $0.043^{*}$ & $0.040^{*}$ & 0.669 & 0.193 \\
\hline Yes & 3 & 90.7 & 22 & 66.6 & 26 & 83.9 & & & & \\
\hline No & 29 &
\end{tabular}

Note: Data are presented as numbers $(n)$ and percent (\%). $p$-Value $<0.05$ is considered significant. P1: comparison between I and II; P2: comparison between I and III; P3: comparison between II and III.

*Statistically significant.

The data from our study showed a significant increase in RBCs and HCT values posttherapy in group I and group III compared with group II.

We suggest that this increase in RBC count might be a consequence of initial bone marrow response (erythroid hyperplasia) that occurred 36 to 48 hours after treatment. This associated increases in RBC count with the increase in HB levels, ARC count, and improvement in iron parameters in response to treatment all are suggestive that ID state starts being treated.

This agreed with other studies that reported a significant increase in RBC and HCT values. ${ }^{11,13}$ This improvement is attributed to improvement in serum iron concentration that was reflected by better hematogenesis and more RBC production. These speculations are supported by the fact that reticulocyte count was found to be improved posttherapy in our studied cases in the three groups compared with pretherapy, but the increase in both groups I and III was statistically higher than group II, with no statistically significant difference between group I and group III. The increase in the reticulocyte count is also attributed to bone marrow hyperactivity in response to treatment.

ARC counts (\%) are useful in the diagnosis of ID and IDA, in both children and newborns, and they are reliable and early predictors of response to oral iron therapy. Our result was in accordance with that of Russo et al, who concluded in their data that efficacy of oral iron therapy is indicated by an increase of reticulocyte count as early as 3 days after the start of therapy, while improvement of $\mathrm{Hb}$ concentration was detected later. So, our results support that LF treatment improves iron delivery to bone marrow in patients with IDA that reflected better hematogenesis irrespective of combination with iron therapy. ${ }^{14}$

MCV and MCH showed a significant increase in group I and group III compared with group II. The significant increase in mean MCV and $\mathrm{MCH}$ values posttherapy was in agreement with another study which reported a rise in mean MCV and $\mathrm{MCH}$ posttherapy. ${ }^{15}$ This improvement indicates not only better hematogenesis but also more perfection in RBC formation to correct the well-known microcytosis and anisocytosis that occur in IDA. 
The data from our study showed a significant decrease in white blood cells in group I compared with group II, but there was no statistically significant difference between group I and group III nor between group II and group III. This can be attributed to the fact that LF, together with secretory immunoglobulin A and defensins, is part of the innate system designed to achieve microbial homeostasis. ${ }^{16} \mathrm{LF}$ is a multifunctional molecule by nature because of its high affinity for ferric iron, which deprives microbes from the free iron necessary for their growth and of its ability to interact with microbial and target host cell surfaces. ${ }^{17}$ Many in vitro studies previously reported the growth-promoting (bifidogenic) effects of LF on bifid bacteria, gram-positive anaerobic bacteria that naturally colonize the human intestinal tract. These bacteria are considered to exert beneficial effects on human health by preventing infections or modulating immune responses and this may be the cause of decreased white blood cells. ${ }^{18}$

Adding LF to the treatment with oral iron (group 3) improved compliance in itself, which is important and supportive of the conclusion. It is also important as it provides an indication of compliance, particularly for group II.

Again, children who received oral bovine LF reported fewer adverse effects than those who received OFS. These results can be attributed to the well-known side effects of iron therapy, such as constipation or diarrhea, anorexia, and gastric upsets, which can interfere with the continuation of treatment. On the other hand, LF as a natural component present in milk represents an ideal nutraceutical product both for its economic production and for its tolerance after ingestion. Taruni also reported the same results as ours. ${ }^{12}$ He found that LF was well tolerated with little side effects.

\section{Conclusion}

It can be concluded that oral bovine LF is more effective in treating ID and IDA compared with OFS with clinical benefits of fewer side effects and better patient compliance. No significant improvement in iron and hematological parameters was noted on the addition of OFS to oral LF, though the combination demonstrated a lower incidence of ADRs.

\section{Authors' Contributions}

All authors read and approved the final manuscript. O.M.E. designed the study, evaluated the patients, collected, analyzed, and interpreted the data. O.M.E., A.G.A., and A.A.S. contributed to the conception and design of the study, literature search, and manuscript review. M.A.D. contributed to the biochemical laboratory assessment, interpretation of data analysis, manuscript writing, and review. All authors helped in revising and approving the manuscript.

\section{Ethical Approval and Consent to Participate}

The study was approved by the Ethics Committee of the Faculty of Medicine, Assiut University (IRB no: 17100915). Verbal informed consents were taken from parents with explanation of benefits of the study, risks expected, and suggested treatment for each case. As the manuscript does not contain any kind of content which can reveal patient's identity, so written informed consent was not taken from the guardians.

\section{Funding}

None.

Conflict of Interest

None declared.

\section{References}

1 Özdemir N. Iron deficiency anemia from diagnosis to treatment in children. Turk Pediatri Ars 2015;50(01):11-19

2 Al Ghwass MM, Halawa EF, Sabry SM, Ahmed D. Iron deficiency anemia in an Egyptian pediatric population: a cross-sectional study. Ann Afr Med 2015;14(01):25-31

3 Roganović J, Starinac K. 2018. Iron deficiency Anemia in children, In: Jesmine Khan, eds. Current Topics of Anemia. Croatia: InTech Open, Janeza Trdine; 47-71

4 Camaschella C. Iron deficiency: new insights into diagnosis and treatment. Hematology (Am Soc Hematol Educ Program) 2015; 2015(01):8-13

5 Jiang R, Lönnerdal B. Cloning and characterization of the human lactoferrin receptor gene promoter. Biometals 2018;31(03):357-368

6 Rosa L, Cutone A, Lepanto MS, Paesano R, Valenti P. Lactoferrin: a natural glycoprotein involved in iron and inflammatory homeostasis. Int J Mol Sci 2017;18(09):1985

7 Superti F. Lactoferrin from bovine milk: a protective companion for life. Nutrients 2020;12(09):2562

8 Wang B, Timilsena YP, Blanch E, Adhikari B. Lactoferrin: structure, function, denaturation and digestion. Crit Rev Food Sci Nutr 2019; 59(04):580-596

9 Russo G, Guardabasso V, Romano F, et al. Monitoring oral iron therapy in children with iron deficiency anemia: an observational, prospective, multicenter study of AIEOP patients (Associazione Italiana Emato-Oncologia Pediatrica). Ann Hematol 2020;99(03):413-420

10 Adamson JW. Iron deficiency and other hypoproliferative anemias. In: Fauci AS, Braunwald E, Kasper DL et al, eds. Harrison's Principles of Internal Medicine. New York, NY: McGraw-Hill; 2008:628-634

11 El-Khawaga A, Abdelmaksoud H. Effect of lactoferrin supplementation on iron deficiency anemia in primary school children. Int J Med Arts 2019;1(01):48-52

12 Taruni R. A Comparative Study to Evaluate the Efficacy of Oral Lactoferrin Fortified Bovine Colostrum with Oral Iron in the treatment of Iron Deficiency Anemia [Doctoral dissertation]. Chennai, India: Kilpauk Medical College; 2015

13 Paesano R, Berlutti F, Pietropaoli M, Goolsbee W, Pacifici E, Valenti P. Lactoferrin efficacy versus ferrous sulfate in curing iron disorders in pregnant and non-pregnant women. Int J Immunopathol Pharmacol 2010;23(02):577-587

14 Parodi E, Giraudo MT, Davitto M, et al. Reticulocyte parameters: markers of early response to oral treatment in children with severe iron-deficiency anemia. J Pediatr Hematol Oncol 2012;34 (06):e249-e252

15 Saha L, Pandhi P, Gopalan S, Malhotra S, Saha PK. Comparison of efficacy, tolerability, and cost of iron polymaltose complex with ferrous sulphate in the treatment of iron deficiency anemia in pregnant women. MedGenMed 2007;9(01):1

16 Embleton ND, Berrington JE, McGuire W, Stewart CJ, Cummings SP. Lactoferrin: antimicrobial activity and therapeutic potential. Semin Fetal Neonatal Med 2013;18(03):143-149

17 Wada Y, Lönnerdal B. Bioactive peptides derived from human milk proteins-mechanisms of action.J Nutr Biochem 2014;25(05):503-514

18 Oda $\mathrm{H}$, Wakabayashi $\mathrm{H}$, Yamauchi K, Abe F. Lactoferrin and bifidobacteria. Biometals 2014;27(05):915-922 